\title{
Generalized Bloch Wave Analysis for Fiber and Waveguide Gratings
}

\author{
Eva Peral and José Capmany, Senior Member, IEEE
}

\begin{abstract}
We have developed a generalized Bloch wave approach for the analysis of aperiodic gratings. This method yields both a macroscopic (i.e., reflection or transmission coefficient) as well as a microscopic (i.e., dispersion diagram and microstructure of the propagating internal field) characterization of fiber and waveguide aperiodic gratings.
\end{abstract}

Index Terms-Bloch waves, gratings.

\section{INTRODUCTION}

$\mathbf{T}$ HE recent advances in fiber and integrated optics fabrication methods have provided the possibility of writing periodic and aperiodic gratings in both technologies in a costeffective way. Especially remarkable have been the achievements accomplished in the fabrication of gratings exploiting the photosensitivity of the core material in standard optical fibers which in the last few years have rendered up the possibility of implementing corrugated structures with almost any envisaged grating period (uniform or chirped) and coupling coefficient apodization [1]-[5].

The application of these structures and their related devices are numerous and include among others, distributed feedback (DFB) [6] and all fiber lasers, optical filters for wavelength division multiplexing (WDM) systems [7], pulse compression, dispersion compensation in both digital [8], [9] and analog [10] optical communications, and optical sensors [11].

The theoretical aspects related to electromagnetic wave propagation in fiber and waveguide gratings have attracted the interest of researchers well before their practical availability and work in this field spans over the last two decades. Several methods for the analysis of the field propagation in corrugated structures have been developed [12]-[24]. Perhaps the most widely used has been the coupled mode theory (CMT) [12], where the counterpropagating fields inside the grating structure obtained by convenient perturbation of the fields in the unperturbed waveguide are related by coupled differential equations. This method, initially developed for uniform gratings, was extended to aperiodic structures by Kogelnik [13] and yields the structure field reflection and transmission coefficients. The CMT approach usually involves the numerical solution of two coupled differential equations, since analytic solutions are only possible for the uniform grating. Matrix methods [14], [15]

Manuscript received January 14, 1997; revised May 2, 1997.

E. Peral is with the California Institute of Technology, Pasadena, CA 91125 USA.

J. Capmany is with the Departamento de Comunicaciones, ETSI Telecomunicación, Camino de Vera s/n, 46071, Valencia, Spain.

Publisher Item Identifier S 0733-8724(97)05937-9. which require as well numerical treatment have also been developed for the purpose of grating analysis. Noteworthy approaches have been the effective index method (EIM) [14] and the transfer matrix method (TMM) [15]. In the former, the grating is divided into sections, the length of each one being much smaller than the smallest value of the corrugation period and the fields are computed inside each section under the hypothesis that the refractive index remains constant using the effective index method of integrated optics. Fields in each section are impedance matched to those of its preceding and succeeding sections yielding a matrix relationship between the fields at the left and right part of each section, the overall structure is characterized by a global matrix obtained from the multiplication of the individual matrices of its sections. The EIM approach is especially suitable for integrated optic gratings, where the maximum structure lengths are in the order of some millimeters, but may require excessive computation time for fiber gratings, where the structure lengths are typically in the $\mathrm{cm}$ range.

In the case of the TMM [15] the grating is divided into sections, where the length of each one is much bigger than the biggest period of the corrugation and where the index variation inside each one is such that they can be considered uniform gratings (with different parameters for each one). Each section is described therefore by a transfer matrix corresponding to an uniform grating [12], [23], and the overall structure is characterized by a global matrix obtained as the product of the individual matrices. This approach is well suited for periodic and aperiodic gratings, and is especially recommendable for long gratings, such as those fabricated in fibers.

Other less known or employed methods have been developed and published in the literature. For instance, [16] presents an extension of the well-known Rouard's method employed in thin film design to the characterization of waveguide gratings, and [17] develops an elegant discrete-time approach based on a digital signal processing formulation for the analysis of periodic gratings. Other methods based on more fundamental physics-oriented approaches such as the WKB [18], Hamiltonian [19], and variational [20] principles are also available in the literature.

In the case of uniform waveguide gratings the former approaches provide only an approximate solution, since the exact solution is obtained using the Bloch theory [21]-[23]. Bloch waves are the eigenmodes of periodic media in the same way as plane waves are the natural modes of free space propagation. Furthermore, as pointed elsewhere [23] the field propagation in waveguide gratings is difficult to interpret without an 
understanding of its dispersion and microstructure. Whereas the coupled mode and the rest of methods described above do provide an overall description of the grating operation with a highly complex and difficult to interpret picture of the internal field, the Bloch wave approach has the added bonus that it provides a clear picture of the field dispersion and microstructure.

In the case of aperiodic gratings, it would be desirable to dispose of an approach with similar characteristics in terms of simple description of field dispersion and microstructure. In this case an extension of the Bloch wave approach does lead to an approximate solution, which has to be computed numerically. The advantage is that while other methods still require this numerical computation and provide also approximate solutions, the generalized Bloch wave analysis developed in this paper benefits as well from a powerful description of the physics involved in the field propagation.

The objective of this paper is therefore to present the derivation of a generalized Bloch wave analysis, suitable for aperiodic waveguide gratings. The paper is structured as follows. Section II presents the formulation of the generalized Bloch wave problem for aperiodic gratings that leads to a fundamental eigenvalue system. The specialized treatment of this eigenvalue system is then considered both for periodic (not necessarily sinusoidal) and for aperiodic gratings separately. In the later case a finite element technique is used. In Section III we apply the generalized method to the solution of specific cases of practical interest, that include, uniform, sampled, and linearly chirped gratings. The results show that both macroscopic (reflection coefficient) as well as microscopic (field dispersion diagrams) characteristics are available using this approach.

\section{Formulation OF THE Generalized Bloch WaVe Problem}

The perturbation in the grating can be expressed in a general way as [12]

$$
\varepsilon(x, y, z)=\varepsilon_{0}(x, y)+\Delta \varepsilon(x, y, z) .
$$

It can be shown that by writing solution to the wave equation of the TE modes as

$$
E_{v}(x, y, z)=a(z) e_{v}(x, y)
$$

where $v$ is the mode number, $a(z)$ is the slowly varying field amplitude and $e_{v}(x, y)$ is the modal field in the unperturbed waveguide, the wave equation reduces to

$$
\frac{d^{2}}{d z^{2}} a(z)+k_{0}^{2} \varepsilon(z) a(z)=0
$$

where $N(z)=\sqrt{\varepsilon(z)}$ is the waveguide local modal index of the unperturbed waveguide and $k_{0}=\omega \sqrt{\mu_{0} \varepsilon_{0}}$ is the freespace wave vector. This equation turns out to be identical to the wave equation describing reflection of TE plane waves in a inhomogeneous medium. A similar expression can be found for TM modes.
For the case of an aperiodic grating, the local modal index can be modeled by a quasisinusoidal function [13], thus

$$
\varepsilon(z)=\bar{\varepsilon}(z)+\Delta \varepsilon(z) \cos [G z+\theta(z)]
$$

where $G$ is the mean wave vector of the perturbation and $\theta(z)$ accounts for spatial deviations from the mean period. It is worth noting that the first term in the right-hand side (RHS) of (2.4) that represents the slowly varying part of the modal index is often disregarded, but we will keep it thorough the entire derivation. On the other hand, the more general case where the perturbation is not assumed to vary rapidly as $\cos (G z)$ is straightforwardly analyzed following the same procedure as that presented here.

In order to obtain the dispersion map of the modal field we perform a transformation and express the spatial spectrum of $a(z)$ in terms of wave vector. Let $A(k)$ be defined as

$$
A(k)=\frac{1}{2 \pi} \int_{-\infty}^{\infty} a(z) \exp (j k z) d z .
$$

Since the wave vector can be complex in the case of evanescent waves, the expression above is actually the bilateral Laplace transform of $a(z)$. The region of convergence of this transform will be a horizontal strip $\gamma_{1}<\operatorname{Imag}\{k\}<\gamma_{2}$ in the complex wave vector plane. The inverse formula can be readily obtained by realizing that $A\left(k=k_{r}+i k_{i}\right)$ is the inverse Fourier transform of $a(z) \exp \left(-k_{i} z\right)$. Thus

$$
\begin{aligned}
a(z) & =\exp \left(k_{i} z\right) \int_{-\infty}^{\infty} A\left(k_{r}+i k_{i}\right) \exp \left(-j k_{r} z\right) d k_{r} \\
& =\int_{-\infty+j k_{i}}^{\infty+j k_{i}} A(k) \exp (-j k z) d k
\end{aligned}
$$

Similarly, we define

$$
\varepsilon(z)=\int_{-\infty}^{\infty} \tilde{\varepsilon}\left(k_{r}\right) \exp \left(-j k_{r} z\right) d k_{r} .
$$

For example, the spectral representation of the source or perturbation will be given by an inverse Fourier transform of $\varepsilon(z)$ over the real axis of the complex $k$ plane.

By introducing the spectral representations of $a(z)$ and $\varepsilon(z)$ in the wave equation (2.3), we end up with

$$
\begin{aligned}
& \int_{-\infty+j k_{i}}^{\infty+j k_{i}}-k^{2} A(k) \exp (-j k z) d k+k_{0}^{2} \cdot \exp \left(k_{i} z\right) \\
& \int_{-\infty}^{\infty} A\left(k_{r}+j k_{i}\right) \exp \left(-j k_{r} z\right) d k_{r} \\
& \int_{-\infty}^{\infty} \varepsilon\left(k_{r}\right) \cdot \exp \left(-j k_{r} z\right) d k_{r}=0 .
\end{aligned}
$$

A simple change of variable yields the following equation:

$$
\begin{aligned}
& \int_{-\infty+j k_{i}}^{\infty+j k_{i}}\left[-k^{2} A(k)+k_{0}^{2} \int_{-\infty}^{\infty} A\left(k-k_{r}^{\prime}\right) \varepsilon\left(k_{r}^{\prime}\right) d k_{r}^{\prime}\right] \\
& \cdot \exp (-j k z) d k=0
\end{aligned}
$$

that will be satisfied if

$$
-K^{2} A(K)+k_{0}^{2} \int_{-\infty}^{\infty} A\left(K-k_{r}^{\prime}\right) \varepsilon\left(k_{r}^{\prime}\right) d k_{r}^{\prime}=0 .
$$


Note that the variable $k$ has been replaced by $K$ to express the fact that, given the frequency $\omega$, the integral equation above has solution for a set of eigenvalues $K$. Incidentally this is one of the objectives of the present analysis, i.e., to obtain a dispersion relation of the form $\omega=\omega(K)$ [or similarly $\left.k_{0}=k_{0}(K)\right]$ that permits the representation of dispersion maps. In the case of an aperiodic grating, the spectrum of $\varepsilon(z)$ is continuous and therefore there is an infinite continuum of eigenvalues. On the contrary, for finite length periodic perturbations, i.e., when $\varepsilon_{-1}(z), \varepsilon_{0}(z)$, and $\varepsilon_{1}(z)$ are periodic functions (not necessarily sinusoidal), an infinite set of discrete eigenvalues is found.

On substitution of the general expression for an aperiodic perturbation (2.4) into (2.7), we obtain

$$
\begin{aligned}
\tilde{\varepsilon}\left(k_{r}\right)= & \varepsilon_{-1}\left(k_{r}\right) * \delta\left(k_{r}+G\right)+\varepsilon_{0}\left(k_{r}\right) \\
& +\varepsilon_{1}\left(k_{r}\right) * \delta\left(k_{r}-G\right) .
\end{aligned}
$$

In the case of a lossless medium with real permittivity, then $\varepsilon_{-1}\left(k_{r}\right)=\varepsilon_{1}^{*}\left(k_{r}\right)$, where the star denotes complex conjugate. We further assume that $\bar{\varepsilon}(z)$ and $\Delta \varepsilon(z)$, as well as $a(z)$, are slowly varying functions with a finite spatial spectrum bandwidth. Thus we can clearly distinguish three spectral components centered, respectively at $k=-G, k=0$, and $k=$ $G$. Moreover, the $k$ spectrum can be divided into two sets of eigenvalues, either continuous or discrete, one corresponding to copropagating waves and the other to counterpropagating waves. Thus, (2.10) can be expressed as

$$
\begin{aligned}
-K^{2} A(K) & +k_{0}^{2} \\
& \cdot \int_{-\infty}^{\infty} A\left(K-k_{r}^{\prime}\right) \varepsilon_{0}\left(k_{r}^{\prime}\right) d k_{r}^{\prime}+k_{0}^{2} \\
& \cdot \int_{-\infty}^{\infty} A\left(K+G-k_{r}^{\prime}\right) \varepsilon_{-1}\left(k_{r}^{\prime}\right) d k_{r}^{\prime}+k_{0}^{2} \\
& \cdot \int_{-\infty}^{\infty} A\left(K-G-k_{r}^{\prime}\right) \varepsilon_{1}\left(k_{r}^{\prime}\right) d k_{r}^{\prime}=0
\end{aligned}
$$

If a forward propagating wave is incident in the grating structure with initial wave vector $k_{\text {in }} \cong G / 2$, two spectral regions are resonantly coupled, namely those centered at $k=0$ and $k=G$ or equivalently $A(k-G)$ and $A(k)$. On the other hand it is known [24] that the real part of the wave vector in the grating will also be around $G / 2$ and in order to simplify the notation we introduce a new parameter $\gamma=K-G / 2$. Using (2.12), we can obtain two coupled equations that only maintain the phase matched terms and can be expressed in a matrix fashion as

$$
\begin{gathered}
\left\{\gamma^{2} \bar{I}+G \gamma I+\left[\begin{array}{cc}
\left(\frac{G}{2}\right)^{2}-2 k_{0} \hat{q}_{0} & -2 k_{0} \hat{q}_{1} \\
2 k_{0} \hat{q}_{1}^{*} & -\left(\frac{G}{2}\right)^{2}+2 k_{0} \hat{q}_{0}
\end{array}\right]\right\} \\
\cdot\left[\begin{array}{c}
A(K) \\
A(K-G)
\end{array}\right]=0 .
\end{gathered}
$$

Here $I$ is the identity matrix, $\bar{I}$ is the matrix $\bar{I}=\left[\begin{array}{rr}1 & 0 \\ 0 & -1\end{array}\right]$ and the operator $\hat{q}_{i}$ is given by:

$$
\hat{q}_{i} A(K)=\int_{-B}^{B} q_{i}\left(k_{r}^{\prime}\right) A\left(K-k_{r}^{\prime}\right) d k_{r}^{\prime}
$$

where $q_{i}\left(k_{r}\right)=\left(k_{0} / 2\right) \varepsilon_{i}\left(k_{r}\right)$ is the spectral representation of the coupling coefficient. In writing (2.14), we have taken into account the aforementioned fact that the spatial content of $a(z), \bar{\varepsilon}(z)$, and $\Delta \varepsilon(z)$ extends only over a limited portion of the wavevector spectrum, namely, $[-B, B]$.

Equation (2.13) takes the form of a generalized eigenvalue system that can be numerically solved. Nevertheless, in the usual case of weak perturbation, within which the approximate wave equation (2.3) holds, we can assume $|\gamma| / G \ll 1$, and neglect second-order terms. This yields a simple eigenvalue problem that requires less computational effort. If we further consider $\varepsilon_{0}\left(k_{r}\right)=\varepsilon_{0} \delta\left(k_{r}\right)$, i.e., no low spatial frequency variation of the modal refractive index, and study incident fields near the Bragg frequency, i.e., $\delta=k_{0}-G / 2 \ll G$, we obtain

$$
\left(\left[\begin{array}{cr}
\delta & \hat{q}_{1} \\
-\hat{q}_{1}^{*} & -\delta
\end{array}\right]\right)\left[\begin{array}{c}
A(K) \\
A(K-G)
\end{array}\right]=\gamma\left[\begin{array}{c}
A(K) \\
A(K-G)
\end{array}\right]
$$

In general, the numerical solution of both eigensystems (2.13) and (2.15) is not possible to obtain analytically, but numerical techniques are easily implemented. Subsequently, we tackle the periodic and aperiodic case separately.

\section{A. Periodic Perturbation}

In the case of a periodic perturbation (not necessarily sinusoidal), the spectrum of the modal index consists of trains of delta functions spaced by $g_{i}$ where $2 \pi / g_{i}$ is the period of the slowly varying $\varepsilon_{i}(z)$ for $i$ equal to $-1,0$, and 1 , and centered, respectively, at $k=-G, 0$, and $G$. For the sake of simplicity we assume all the periods to be the same, and thus the spectral components resonantly coupled are $A(K-G+m g)$ and $A(K+m g)$ for $m$ an integer ranging from $-N$ to $N$ where $B=N g$ is the spatial bandwidth considered. Now the integral system (2.13) has been reduced to an eigensystem $(4 N \times 4 N)$ that, for the simpler example yielding (2.15), is shown in (2.16) at the bottom of the next page where $q_{m}$ are the weights of the delta functions at $k=G-m g$ and, in order to simplify the notation, we have set $A_{m}=$ $A(K-m g)$ and $B_{m}=A(K-G-m g)$. The eigenvector corresponding to the eigenvalue $\gamma_{m}$ will be represented as

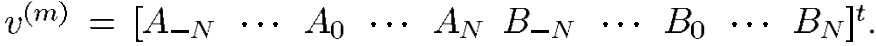
The resultant field will consist in a linear combination of the solution eigenvectors, or in other words, a summation over co- and counterpropagating Bloch waves with different propagation constants and weights

$$
\begin{aligned}
A(k)= & \sum_{m=1}^{4 N+2} a_{m}\left\{A_{-N}^{(m)} \delta\left[k-\left(\frac{G}{2}+\gamma_{m}-N g\right)\right]+\cdots\right. \\
& +A_{N}^{(m)} \delta\left[k-\left(\frac{G}{2}+\gamma_{m}+N g\right)\right] \\
& +B_{-N}^{(m)} \delta\left[k-\left(\frac{G}{2}+\gamma_{m}-N g\right)\right]+\cdots \\
& \left.+B_{N}^{(m)} \delta\left[k-\left(\frac{G}{2}+\gamma_{m}+N g\right)\right]\right\}
\end{aligned}
$$




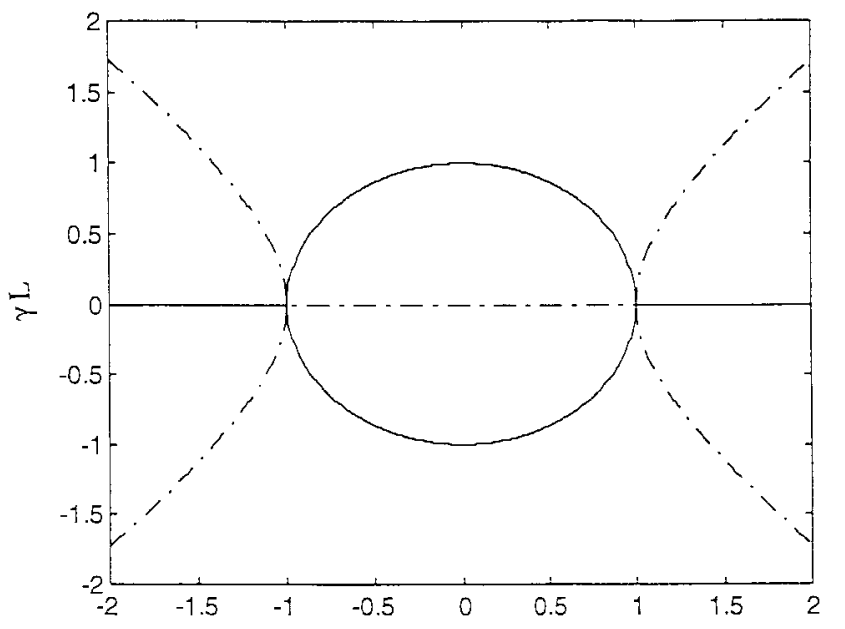

$\delta \mathrm{L}$

Fig. 1. Dispersion map of the uniform grating: real (dashed) and imaginary part (solid).

that, when transformed back into the expression for the field, yields

$$
\begin{aligned}
E^{+}(z)= & \exp \left(-j \frac{G}{2} z\right) \sum_{m=1}^{4 N+2} a_{m}\{ \\
& A_{-N}^{(m)} \exp \left[-j\left(\gamma_{m}-N g\right) z\right]+\cdots+ \\
& \left.A_{N}^{(m)} \exp \left[-j\left(\gamma_{m}+N g\right) z\right]\right\}
\end{aligned}
$$

for the forward field and

$$
\begin{aligned}
E^{-}(z)= & \exp \left(-j \frac{G}{2} z\right) \sum_{m=1}^{4 N+2} a_{m}\{ \\
& B_{-N}^{(m)} \exp \left[-j\left(\gamma_{m}-N g\right) z\right]+\cdots+ \\
& \left.B_{N}^{(m)} \exp \left[-j\left(\gamma_{m}+N g\right) z\right]\right\}
\end{aligned}
$$

for the backward field.

Application of the boundary conditions at $z=z_{0}$ and $z=z_{f}$ yield the coefficients of the series expansion $a_{m}$. For a forward wave impinging on the grating at $z=z_{0}$ and no backward wave entering the grating at $z=z_{f}$, this condition translates into

$$
\begin{aligned}
& {\left[\begin{array}{ccccc} 
& \vdots & & & \vdots \\
\exp \left(-j \gamma_{1} z_{0}\right) & A^{(1)} & \cdots & \exp \left(-j \gamma_{M} z_{0}\right) & A^{(M)} \\
& \vdots & & & \vdots \\
\exp \left(-j \gamma_{1} z_{f}\right) & B^{(1)} & \cdots & \exp \left(-j \gamma_{M} z_{f}\right) & B^{(M)} \\
& \vdots & & & \vdots
\end{array}\right]} \\
& \cdot\left(\begin{array}{c}
a_{1} \\
a_{2} \\
\vdots \\
a_{M}
\end{array}\right)=\left(\begin{array}{c}
0 \\
\vdots \\
1 \\
\vdots \\
0 \\
0 \\
\vdots \\
0
\end{array}\right)
\end{aligned}
$$

where the eigenvector $(m)$ is split into two block vectors $v^{(m)}=\left[\begin{array}{c}A^{(m)} \\ B^{(m)}\end{array}\right]$, and $M=4 N+2$ is the total number of eigenvalues. The reflection coefficient is readily obtained as $r(\delta)=E^{-}\left(z_{0}\right) / E^{+}\left(z_{0}\right)$.

\section{B. Aperiodic Perturbation}

We have previously argued that an aperiodic perturbation would result in a continuum of eigenvalues and the field would be obtained from an integral over all of them. However this is not easily tractable analytically apart from for a few specific cases and a numerical solution turns out to be necessary. The best way to tackle this problem is by using a finite element approach, where the unknown functions are first discredited and then interpolated. We can employ first-order interpolation

$$
A\left(K-k_{r}\right)=\sum_{m=-N}^{N-1} N_{1}\left(\frac{k_{r}}{g_{m}}\right) A_{m}+N_{2}\left(\frac{k_{r}}{g_{m}}\right) A_{m+1}
$$

$$
\left(\begin{array}{ccccccccccc}
\delta-N g & & 0 & \cdots & 0 & q_{0} & \cdots & q_{N} & & 0 \\
& \ddots & & & \vdots & \vdots & & & & \vdots \\
0 & & \delta & & 0 & q_{-N} & \cdots & q_{0} & \cdots & q_{N} \\
\vdots & & & \ddots & \vdots & & & & & \vdots \\
0 & \cdots & 0 & \cdots & \delta+N g & 0 & \cdots & q_{-N} & \cdots & q_{0} \\
-q_{0}^{*} & \cdots & -q_{N}^{*} & & 0 & -\delta-N g & & 0 & \cdots & 0 \\
\vdots & & & & & & \ddots & & & \vdots \\
-q_{-N}^{*} & \cdots & & \cdots & -q_{N}^{*} & 0 & \cdots & -\delta & \cdots & 0 \\
0 & & & & & \vdots & & & \ddots & \vdots \\
0 & -q_{0}^{*} & \cdots & -q_{N}^{*} & 0 & \cdots & 0 & \cdots & -\delta+N g
\end{array}\right)\left(\begin{array}{c}
A_{-N} \\
\vdots \\
A_{0} \\
\vdots \\
A_{N} \\
B_{-N} \\
\vdots \\
B_{0} \\
\vdots \\
B_{N}
\end{array}\right)=\gamma\left(\begin{array}{c}
A_{-N} \\
\vdots \\
A_{0} \\
\vdots \\
A_{N} \\
B_{-N} \\
\vdots \\
B_{0} \\
\vdots \\
B_{N}
\end{array}\right)
$$




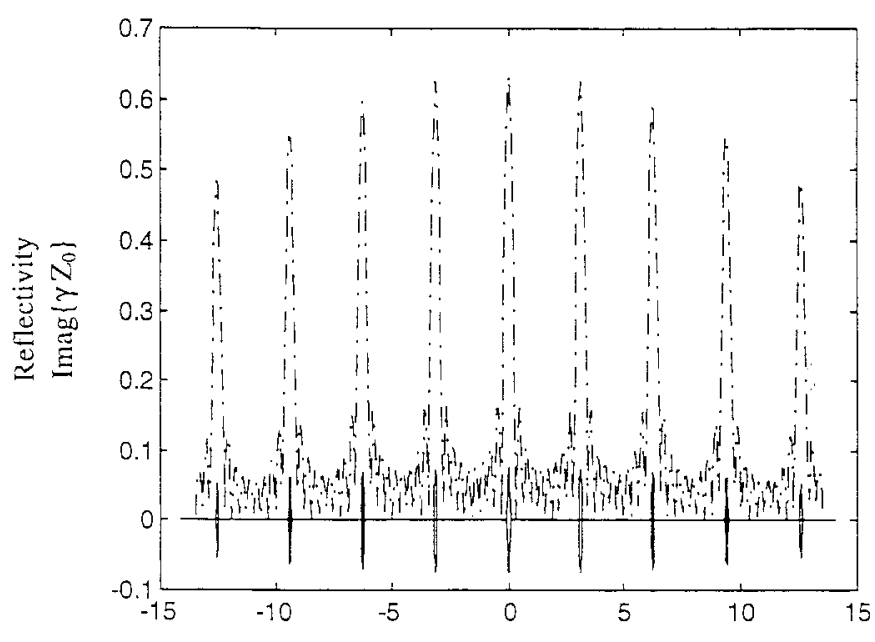

$\delta \mathrm{Z}_{0}$

(a)

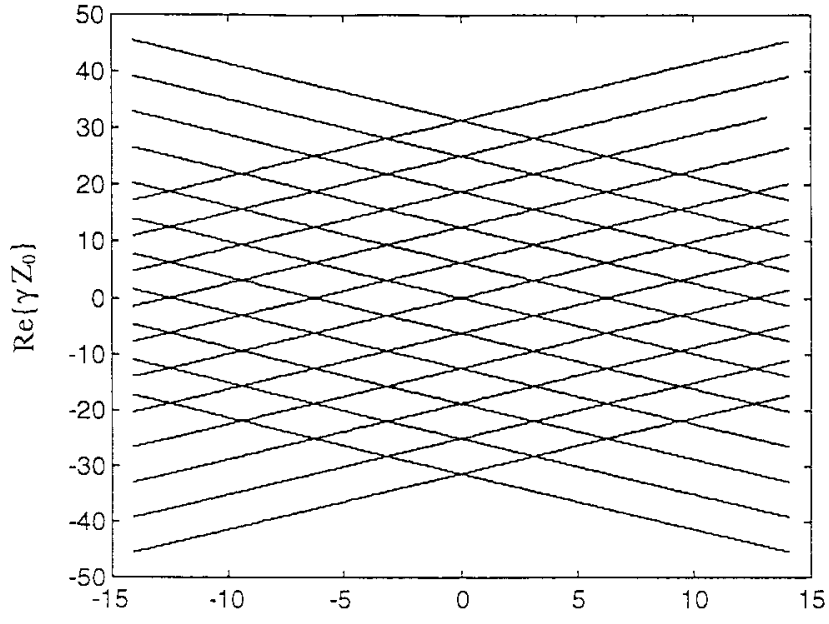

$\delta Z_{0}$

(b)

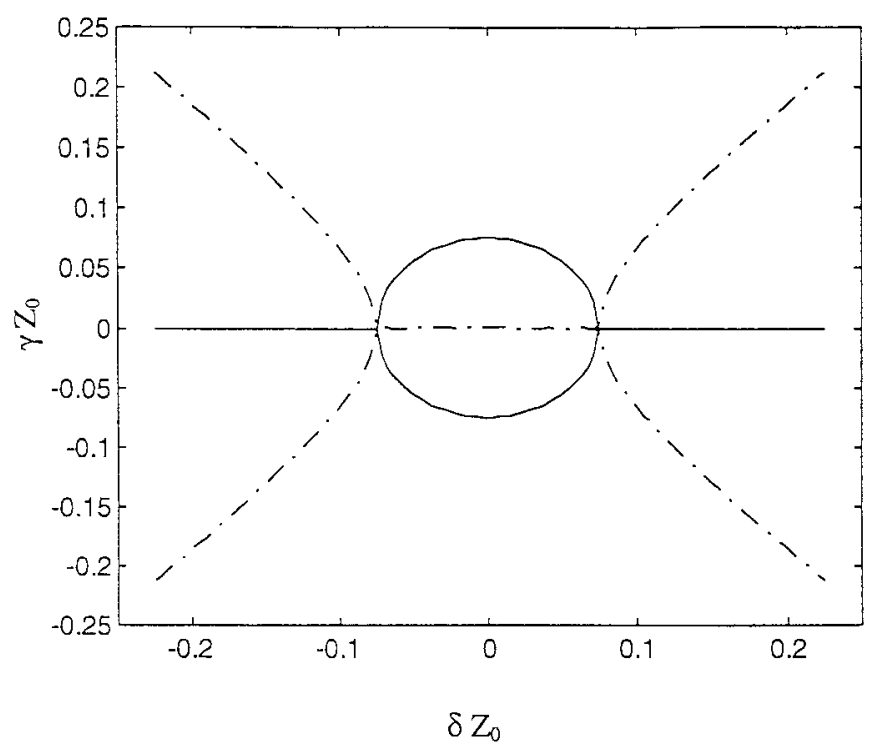

(c)

Fig. 2. Dispersion map of the sampled grating: (a) reflectivity (dashed) and imaginary part (solid), (b) real part, and (c) detail at $\delta=0$ real (dashed) and imaginary part of eigenvalues (solid).

or second-order interpolation

$$
\begin{aligned}
A\left(K-k_{r}\right)= & \sum_{\substack{m=-N \\
\text { meven }}}^{N-1} N_{1}\left(\frac{k_{r}}{g_{m}}\right) A_{m} \\
& +N_{2}\left(\frac{k_{r}}{g_{m}}\right) A_{m+1}+N_{3}\left(\frac{k_{r}}{g_{m}}\right) A_{m+2} .
\end{aligned}
$$

Higher orders of interpolation do not yield better results and are much more complex. Thus, we have $4 N+2$ nodes (either equally or not equally spaced), and we need the same number of equations in order to solve the system. These can be obtained by evaluating (2.10) at the different node locations, i.e., $k=K-m g$. Care must be taken when calculating the integrals, especially for second-order interpolation, because some of the integrals are over different elements for $A\left(K-k_{r}\right)$ and $q\left(k_{r}\right)$. It is important to note that the original continuum has turned into a discrete spectrum. Nevertheless, by using a sufficiently fine interpolation, the main features of the dispersion map can still be obtained. The final eigensystem looks like (2.16) where the terms $q_{i}$ are now the result of the integration.

The expression for the field is given by

$$
\begin{aligned}
& E^{+}(z)=\sum_{m} a_{m} \int_{-\infty+j k_{i}}^{\infty+j k_{i}} A^{(m)}(k) \exp (-j k z) d k \\
& E^{-}(z)=\sum_{m} a_{m} \int_{-\infty+j k_{i}}^{\infty+j k_{i}} A^{(m)}(k-G) \exp (-j k z) d k .
\end{aligned}
$$




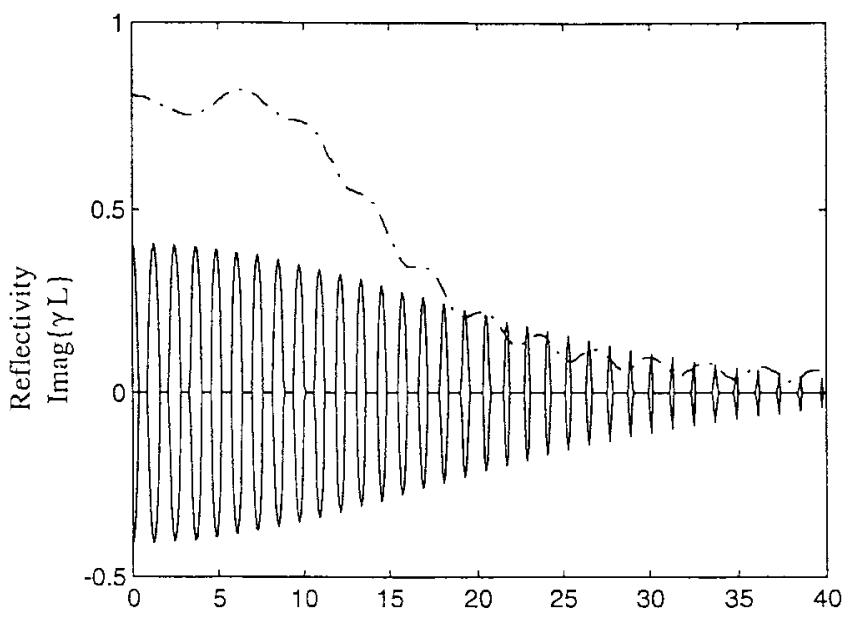

$\delta \mathrm{L}$

(a)

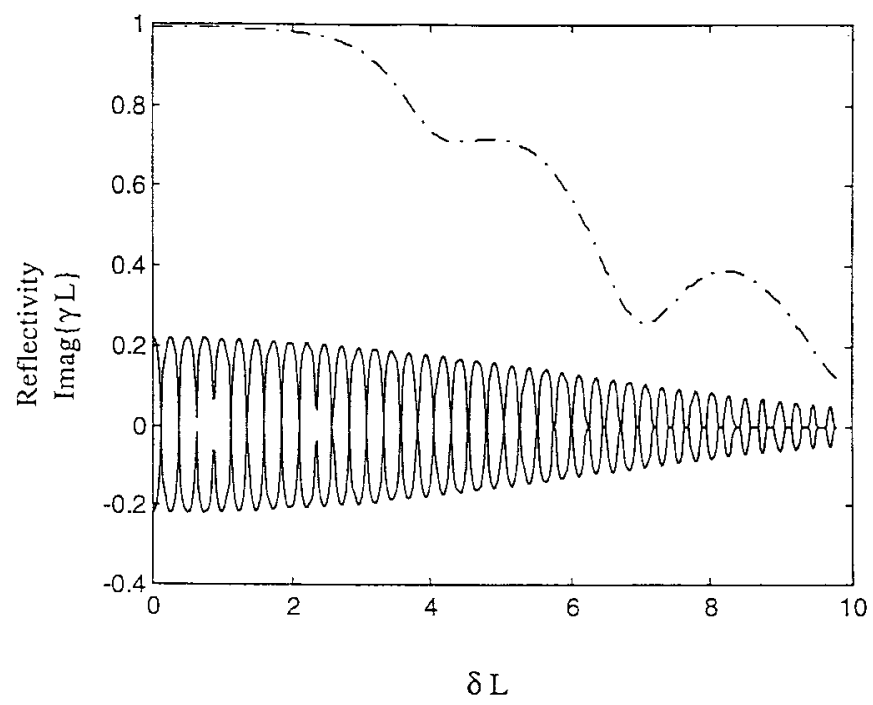

(b)

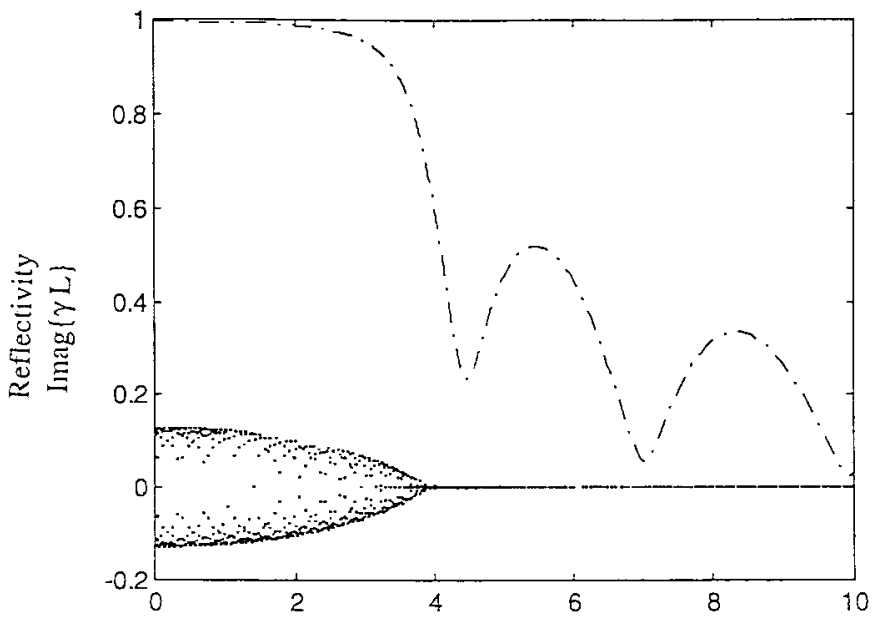

$\delta \mathrm{L}$

(c)

Fig. 3. Linearly chirped grating. Reflectivity (dashed) and imaginary part of dominant eigenvalues (solid) for (a) $F=10 \pi$, (b) $F=2 \pi$, and (c) $F=\pi / 2$ (here only envelope is shown). The dispersion map is symmetric.

By a change of variable this can be transformed into

$$
\begin{aligned}
E^{+}(z)= & \exp \left(-j \frac{G}{2} z\right) \sum_{m} a_{m} \exp \left(-j \gamma_{m} z\right) \\
& \cdot \int_{-\infty}^{\infty} A\left(K-k_{r}\right) \exp \left(j k_{r} z\right) d k_{r}
\end{aligned}
$$

and similarly for $E^{-}(z)$. These integrals can be evaluated from the previously calculated nodes and interpolation functions, whereas the coefficients follow from the boundary conditions as above.

\section{EXAMPLES}

\section{A. Uniform Grating}

Before tackling more complicated problems, let us review the simple case of a uniform grating [23] with constant coupling coefficient $q(z)=q=(\pi / \lambda)(\Delta \varepsilon / 2)$. The dispersion map consists in this case of two eigenvalues $\gamma= \pm \sqrt{\delta^{2}-q^{2}}$. These have been plotted in Fig. 1. We see that for $|\delta|<|q|$ the eigenvalue is imaginary, corresponding to a forbidden band, whereas for $|\delta| \gg|q|$, the eigenvalue tends to the propagation constant in free space for both the forward and backward traveling waves.

\section{B. Sampled Grating}

Sampled Bragg gratings are gratings whose refractive index amplitude is itself periodically modulated [24]. The coupling coefficient can be represented as

$$
q(z)=\kappa_{0} \sum_{n} \Pi\left(\frac{z-n Z_{0}}{Z_{1}}\right) \quad|z|<\frac{L}{2}
$$

where $\Pi(z / Z)$ is a square function centered at the origin and with total width $Z$. The spatial spectrum of the refractive index consists of trains of delta functions spaced by $2 \pi / Z_{0}$ and amplitude $q_{n}=\kappa_{o}\left(Z_{1} / Z_{0}\right)\left[\sin \left(\pi n Z_{1} / Z_{0}\right) /\left(\pi n Z_{1} / Z_{0}\right)\right]$. 
As an example we consider a sampled grating with $Z_{1} / Z_{0}=$ $\frac{1}{9}$, total length $L=10 Z_{0}$ and amplitude $\kappa_{0} Z_{0}=1$. The reflection coefficient has been calculated using the herein presented Bloch wave analysis and the standard coupled wave equations. Both coincide, what constitutes an additional check on the validity of our method. Fig. 2(a) shows the reflectivity together with the imaginary part of the eigenvalues. Since the ratio $Z_{1} / Z_{0}$ is small enough, the reflectivity peaks are spaced far enough that the dispersion map is a superposition of that for the uniform grating, with weights $q_{n}$ at $\delta_{n}=n \pi / Z_{0}$, where the reflectivity peaks. Therefore at each of these frequencies $\delta_{n}$ the eigenvalues have nonzero imaginary part that resembles that of the uniform grating in Fig. 1 where now the radius of the circles is $q_{n}$. The real part of the eigenvalues [see Fig. 2(b)] tends to that corresponding to traveling waves with wave vectors separated by $2 \pi / Z_{0}$ far from the forbidden bands. The dispersion map near a reflectivity peak can be better visualized in Fig. 2(c) for the fundamental harmonic at $\delta_{0}$ where we observe the same features as in Fig. 1.

\section{Linearly Chirped Gratings}

The coupling coefficient of a linearly chirped grating can be expressed as [13]

$$
q(z)=\kappa_{0} \exp \left[i F\left(\frac{z}{L}\right)^{2}\right]
$$

In this case, we are dealing with an aperiodic perturbation, and we can apply the method developed above. In order to find the spatial spectrum of the coupling coefficient, we assume a Gaussian apodization with very large width, so that within the extension of the grating the amplitude can be considered as constant. Although the eigenvalues form a continuum, it turns out that some of them have more weight that others in the sense that the coefficients $a_{m}$ that appear in the field expansion (2.18) are much larger.

We have computed the dispersion map for several values of the chirp parameter $F$ and amplitude $\kappa_{0} L=\pi$. In all cases we observe that the imaginary part of the coupling coefficient consists of circles that extend over a range coincident with maximum reflectivity, what we call extended forbidden band. As $F$ increases, the circles not only extend over a larger range of $\delta$ (the width of the extended forbidden band increases), but are also farther apart and become more distinct (Fig. 3). This provides a physical interpretation of the chirped grating: as the chirp parameter $F$ increases, the structure behaves as a superposition of uniform gratings centered at progressively farther spaced wavelengths. This is the origin of the ripples in the reflection coefficient, that are shallower but have faster oscillation as the value of $F$ is raised. It is also remarkable the absence of dips in the extended forbidden band. On the other hand, for smaller $F$ the dominant eigenvalue follows the envelope of the circles, tending to the dispersion of a single uniform grating. The circles are closer to each other, and the reflectivity is smoother, but only as long as the imaginary part of the eigenvalue is not zero. Outside of the forbidden band, an oscillatory behavior of the reflectivity with narrower dips appear. Finally, the real part of the eigenvalues presents a

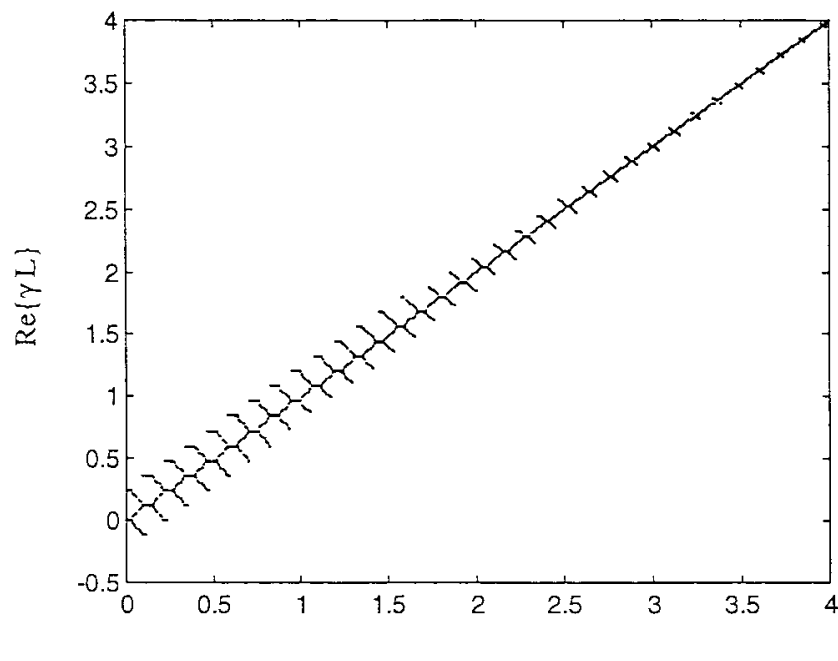

$\delta \mathrm{L}$

Fig. 4. Linearly chirped grating. Real part of dominant eigenvalues for $F=10 \pi$.

lattice-type map, and if we focus on the dominant eigenvalues, we can see that this is in essence the wave vector of the forward traveling wave (Fig. 4).

\section{CONCLUSIONS}

We have developed a generalized Bloch wave approach for the analysis of aperiodic gratings. We believe, that this method will contribute to a better understanding of these structures, since it provides microscopic characterization (i.e., dispersion diagram and microstructure of the propagating internal field), which more conventional methods lack. It can also be applied to the study of nonlinear waveguides, where the knowledge of the forbidden bands is important.

\section{ACKNOWLEDGMENT}

E. Peral is grateful to A. Bäcker for very helpful discussions.

\section{REFERENCES}

[1] G. Meltz, W. W. Morey, and W. H. Glenn, "Formation of Bragg gratings in optical fibers by a transverse holographic method," Opt. Lett., vol. 14, pp. 823-825, 1989.

[2] R. Kashyap, P. F. Mckee, and D. Armes, "UV written grating structures in photosensitive optical fibers using phase-shifted phase masks," Electron. Lett., vol. 30, pp. 1977-1978, 1994.

[3] D. Z. Anderson, V. Mizrahi, T. Erdogan, and A. E. White, "Formation of in-fiber gratings using a diffractive optical element," Electron. Lett., vol. 29, pp. 566-568, 1993.

[4] J. Albert, K. O. Hill, B. Malo, S. Theriault, F. Bilodeau, D. C. Johnson, and L. E. Erickson, "Apodization of the spectral response of fiber Bragg gratings using a phase mask with variable diffraction efficiency," Electron. Lett., vol. 31, pp. 222-225, 1995.

[5] W. Loh, M. J. Cole, M. N. Zervas, S. Barcelos, and R. I. Laming, "Complex grating structures with uniform phase masks based on moving fiber-scan beam technique," Opt. Lett., vol. 20, pp. 2051-2053, 1995.

[6] H. Kogelnik and C. W. Shank, "Coupled wave theory of distributed feedback lasers," J. Appl. Phys., vol. 43, pp. 2327-2335, 1972.

[7] F. Bilodeau, D. C. Johnson, S. Theriault, B. Malo, J. Albert, and K. O. Hill, "An all-fiber dense-wavelength-division multiplexer/demultiplexer using photoimprinted Bragg gratings," IEEE Photon. Technol. Lett., vol. 7, pp. 388-390, 1995.

[8] F. Ouellette, "Dispersion cancellation using linearly chirped Bragg grating filters in optical waveguides," Opt. Lett., vol. 16, pp. 847-849, 1987. 
[9] W. H. Loh, R. I. Laming, X. Gu, M. N. Zervas, M. J. Cole, T. Widdowson, and A. D. Ellis, "10 cm chirped fiber Bragg grating for dispersion compensation at $10 \mathrm{~Gb} / \mathrm{s}$ over $400 \mathrm{~km}$ of nondispersion shifted fiber," Electron. Lett., vol. 31, pp. 2203-2205, 1995.

[10] J. Marti, D. Pastor, M. Tórtola, and J. Capmany, "Optical equalization of dispersion-induced nonlinear distortion in subcarrier systems by employing tapered linearly chirped gratings," Electron. Lett., vol. 32, pp. 236-237, 1996.

[11] M. A. Davis and A. D. Kersey, "Matched-filter interrogation technique for fiber Bragg grating arrays," Electron. Lett., vol. 31, pp. 822-825, 1995.

[12] A. Yariv, "Coupled mode theory for guided-wave optics," IEEE $J$. Quantum Electron., vol. QE-9, pp. 919-933, 1973.

[13] H. Kogelnik, "Filter response of nonuniform almost-periodic structures," Bell Syst. Tech. J., vol. 55, no. 1, pp. 109-126, 1976.

[14] K. A. Winick, "Effective-index method and coupled-mode theory for almost periodic waveguide gratings: A comparison," Appl. Opt., vol. 31, pp. 757-764, 1992.

[15] M. Yamada and K. Sakuda., "Analysis of almost-periodic distributed feedback slab waveguide via a fundamental matrix approach," Appl. Opt., vol. 26, pp. 3474-3478, 1987.

[16] L. A. Weller-Brophy and D. G. Hall, "Analysis of waveguide gratings: Application of Rouard's method," J. Opt. Soc. Amer., vol. A-2, pp. 863-871, 1985.

[17] J. L. Frolik and A. E. Yagle, "An asymmetric discrete-time approach for the design and analysis of periodic waveguide gratings," J. Lightwave Technol., vol. 13, pp. 175-185, 1995.

[18] L. Poladian, "Graphical and WKB analysis of nonuniform Bragg gratings," Phys. Rev. E., vol. 48, pp. 4758-4767, 1993.

[19] T. Hirono and Y. Yoshikuni, "A Hamiltonian formulation for coupledwave equations," IEEE J. Quantum Electron., vol. QE-8, pp. 1751-1755, 1974.

[20] L. Poladian, "Variational technique for nonuniform gratings and distributed feedback lasers," J. Opt. Soc. Amer., vol. A-11, pp. 1846-1853, 1974; also see P. St. J. Russell and T. A. Birks, "A Hamiltonian approach to propagation in chirped and nonuniform Bragg grating structures," in OSA Topical Meeting in Photosensitivity and Quadratic Nonlinearity in Glass Waveguides: Fundamentals and Applications. Portland, OR, 1995.

[21] C. Elachi, "Waves in active and passive periodic structures: A review" Proc. IEEE, vol. 64, pp. 1666-1698, 1976.

[22] A. Yariv and P. Yeh, Optical Waves in Crystals. New York: Wiley, 1984.

[23] P. St. J. Russell, "Bloch wave analysis of dispersion and pulse propagation in pure distributed feedback structures," J. Mod. Opt., vol. 38, pp. 1599-1619, 1991; J. Capmany and P. St. J. Russell, "Erratum," J. Mod. Opt., vol. 41, pp. 163-164, 1994.

[24] V. Jayaraman, Z. M. Chuang, and L. A Coldren, "Theory, design, and performance of extended tuning range semiconductor lasers with sampled gratings," J. Quantum Electron., vol. 29, pp. 1824-1834, 1993.

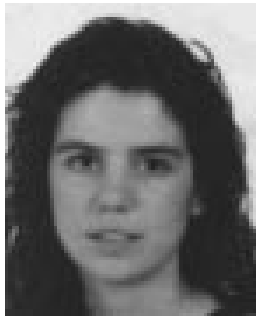

Eva Peral was born on November 23, 1972 in Granada, Spain. She received the degree of Ingeniero Superior de Telecomunicaciones with Honors from the Universidad Politecnica de Valencia (UPV), Spain, in 1995. She began her doctoral studies with the Optical Communications and Photonic Engineering Group in UPV and is completing the Ph.D. degree at the California Institute of Technology, Pasadena, CA.

During 1995, she was a Visiting Student at the University of Southampton, Glasgow, Scotland, and collaborated with the Optoelectronics Group, where she was engaged in integrated optics. She has worked for Philips, Valencia, Spain, and MercedesBenz, Sindelfingen, Germany. Her research interests include fiber gratings, nonlinear phenomena in optical communications, integrated optics, and semiconductor lasers.

Ms. Peral received the National Award for Academic Excellency from the Spanish Ministry of Culture and Education in 1996.

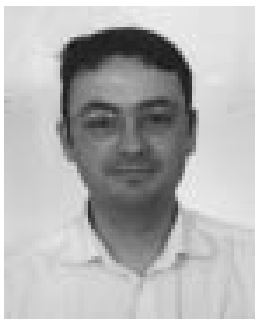

José Capmany (S'88-M'92-SM'96), was born in Madrid, Spain, on December 15, 1962. He received the B.S. and M.S. degrees in telecommunications engineering from the Universidad Politécnica de Madrid (UPM) in 1987 and the Ph.D. degree in telecommunications engineering from the same university in 1991

In 1988, he worked as a Research Assistant at the Photonics Technology Department, UPM. He then moved to the Communications Department of the Universidad Politécnica de Valencia (UPV), where from 1991 to 1996, he was an Assistant Professor and since July 1996, he has been a Full Professor in Optical Communications. From 1991 to 1996, he served as Faculty Vice-Dean for the Telecommunications Engineering School at UPV and started the creation of a research group in optical communications. He has authored or coauthored more than 100 papers in journals and conferences related to photonics and optical communications.

Dr. Capmany is an associate member of IEE and a member of the Technical Committee of the European Conference on Optical Communications (ECOC). $\mathrm{He}$ is the Founder and current Chairman of the IEEE Lasers and Electrooptics Spanish Chapter, and has served as a Reviewer for all the major journals in the field of optical communications. He is a member of the Editorial Board of Fiber \& Integrated Optics and the International Journal of Optoelectronics. 